\title{
Preoperative Albumin-to-Fibrinogen Ratio Predicts Postoperative Pneumonia in Patients with Esophageal Squamous Cell Carcinoma: A Retrospective Study
}

\author{
Peng Zhang \\ Zhengzhou University First Affiliated Hospital \\ Song Zhao ( $\square$ zhaosong@zzu.edu.cn ) \\ Zhengzhou University First Affiliated Hospital
}

\section{Research}

Keywords: Esophageal cancer,postoperative pneumonia,Albumin, Fibrinogen,AFR

Posted Date: December 15th, 2020

DOl: https://doi.org/10.21203/rs.3.rs-127793/v1

License: (c) (i) This work is licensed under a Creative Commons Attribution 4.0 International License. Read Full License 


\section{Abstract}

Background: Postoperative pneumonia is the most common postoperative complication in patients with esophageal cancer. Prediction of postoperative pneumonia by establishing a preoperative physiological function parameter model can help patients make adequate preoperative preparation, reduce treatment costs, and improve prognosis and quality of life. The purpose of this study was to investigate the relationship between albumin, fibrinogen, albumin-to-fibrinogen ratio(AFR) , and other preoperative laboratory tests and postoperative pneumonia in patients with esophageal cancer after esophagectomy.

Methods: Retrospective analysis was performed on 177 consecutive patients who underwent esophagectomy in the Department of Thoracic Surgery, The First Affiliated Hospital of Zhengzhou University from December 2018 to December 2019.Postoperative pneumonia was defined according to the revised Uniform Pneumonia Score(rUPS).Patients were divided into pneumonia and non-pneumonia groups for comparison of baseline data, perioperative indicators, and laboratory examination data. (Receiver operating characteristic)ROC curve analysis was used to evaluate the efficacy, sensitivity and specificity of AFR, and Youden's index was used to calculate the cut-off values of AFR and other laboratory tests data. Univariate and multivariate logistic regression analyses were used to assess the risk factors for postoperative pneumonia

Results: Of the 177 patients, 32 (18\%) developed postoperative pneumonia. The AUC value predicted by AFR using ROC curve analysis was $0.767,65.6 \%$ sensitivity and $83.4 \%$ specificity. Multivariate logistic regression analysis showed that albumin $(P=0.013)$, creatinine $(P=0.01)$, and AFR $(P=0.016)$ were independent risk factors for postoperative pneumonia.

Conclusion: Preoperative AFR can effectively predict the occurrence of postoperative pneumonia in patients with esophageal cancer

\section{Background}

Esophageal cancer is a common malignant tumor worldwide, with a wide range of distribution and a high mortality rate, which brings a great burden of life to people around the world (1). In a study on the causes of death of 508,585 cancer patients worldwide in 2018, the incidence of esophageal cancer ranks the seventh in the world, and the mortality ranks the sixth (2).At present, grade R0 surgical resection for early esophageal cancer or neoadjuvant chemoradiotherapy for locally advanced esophageal cancer (3) is still the main treatment method.In recent years, minimally invasive esophagectomy (MIE) has gradually become the mainstream instead of traditional open surgery.A Randomized Controlled Trial(RCT) showed MIE reduced hospital stay, lung infection, and short-term quality of life compared with traditional open surgery(4), meanwhile several studies have shown that the incidence of pulmonary complications after esophagectomy is still between $20 \%$ and $40 \%$, which is the most common postoperative complication (5).Postoperative pneumonia,as the most common pulmonary complications,seriously affects the prognosis and the quality of life of patients with esophageal cancer (6).Therefore, the establishment of a 
model to predict the possibility of postoperative pneumonia based on preoperative physiological function parameters of patients would be able to help patients make adequate preoperative preparation, reduce treatment costs, improve prognosis and quality of life.

In 2002,T. Nozoel proposed that preoperative nutritional status might predict postoperative complications in patients with esophageal cancer (7).Since then, a variety of nutritional factors, including neutrophils, lymphocytes, C-reactive protein, albumin, fibrinogen and related ratios,even grip strength, have been reported to be independent predictors of postoperative complications and prognosis $(8-10,15)$. Albumin, as an important biochemical index to evaluate nutritional status, has been shown to be associated with postoperative complications and prognosis in patients with a variety of tumors, including esophageal cancer(11-15).Meanwhile, fibrinogen, as an indicator of blood coagulation, has a relatively short half-life and can be used as an acute response protein to reflect systemic inflammation. Guan proposed that low preoperative fibrinogen level may be a potential risk factor for neurological complications after cardiac surgery (16).Recently, a new indicator, albumin-to-fibrinogen ratio(AFR), was proposed in a study to predict postoperative severe complications in elderly patients with gastric cancer (17).Although AFR has been proven to be associated with the prognosis of esophageal cancer (18), it is still unknown whether AFR can predict the occurrence of postoperative pneumonia in patients with esophageal cancer. Therefore, this study was devoted to exploring the relationship between albumin, fibrinogen, AFR and other preoperative laboratory tests and postoperative pneumonia in patients with esophageal cancer.

\section{Patients And Methods}

\section{Patients}

A total of 177 consecutive patients who underwent radical esophagectomy in the Department of Thoracic Surgery, The First Affiliated Hospital of Zhengzhou University from December 2018 to December 2019 were included in this study. 2 cases were were excluded due to tumor invasion, 11 cases were excluded due to lack of data, and 2 cases were excluded due to simultaneous total gastrectomy.All patients underwent preoperative routine examination and evaluation, including complete blood count, biochemical examination of liver and kidney function, blood coagulation examination, pulmonary function examination, b-ultrasound of liver and gallbladder urinary system, b-ultrasound of the heart, chest and upper abdomen enhanced Computed Tomography(CT), skull CT, bone scan, electrocardiogram, gastroscope and barium meal examination.If the patient's general condition allowed, neoadjuvant chemotherapy was performed after discussion in the thoracic department. The chemotherapy drug was cisplatin followed by the next drug: paclitaxel, docetaxel and gemcitabine.One day before the operation, department experts and anesthesiologists discussed and assessed the risks of the operation and preliminarily formulated the operation method.All the patients included in this study underwent mixed minimally invasive esophagectomy including laparoscopy, thoracoscopy and open surgery. The recurrent laryngeal nerve and thoracic duct should be protected during mediastinal stage. The reconstruction of digestive tract was performed by replacing esophagus with tubular stomach, The location of the anastomotic site was determined by neck or chest anastomosis depending on the patient's condition and 
tumor location.Finally, the anastomotic site was wrapped around with greater omentum or high molecular tissue material to prevent anastomotic leakage (19).Postoperative pathology showed that all the 177 patients in this group had squamous cell carcinoma and negative resection margin (R0 resection). The first day after the operation, the patient was examined by bedside chest radiograph.Complete blood count, electrolyte examination,liver and kidney function tests were performed on the $1 \mathrm{st}$ and $3 \mathrm{rd}$ day after the operation.Chest CT was scheduled for the third day after surgery.

All patients who underwent surgery for more than 3 hours received antibiotics, and broad-spectrum antibiotics were routinely applied postoperatively.Tracheal sputum was collected from all patients with suspected pulmonary infection for culture, and the treatment regimen was adjusted on day 2 or 3 according to the patient's clinical manifestations and sputum culture results.All patients received oxygen inhalation, sputum - reducing drug inhalation, injection or analgesics, and intravenous infusion within a few days after surgery. When phlegm is sticky and difficult to cough out, affecting respiration, the patient will be made bedside or tracheobronchial mirror room sputum suction treatment. The retrospective study was approved by the Ethics Committee of the First Affiliated Hospital of Zhengzhou University. Written informed consent was obtained from all eligible patients

\section{Definition of postoperative pneumonia}

In this study, the revised Uniform Pneumonia Score(rUPS) was used to define postoperative pneumonia. The score was based on the following three factors: body temperature $\left({ }^{\circ} \mathrm{C}\right)(\geq 36.1$ and $\leq 38.4=0, \leq 36.0$ or $\geq 38.5=1)$ and white blood cell count $(\times 109 / \mathrm{L})(\geq 4.0$ and $\leq 11.0=0,<4.0$ or $>11.0=1)$ and pulmonary radiological results (chest X-ray and/or CT scan, CT preferred) (no infiltrate=0, diffused(or patchy) infiltrate $=1$, well-circumscribed infiltrate $=2$ ).A sum score of 2 points or higher, of which at least 1 point is assigned due to infiltrative findings on pulmonary radiography, indicates treatment of pneumonia(20).

\section{Data collection}

Preoperative laboratory tests of all patients in this group was completed within one week before surgery.Baseline data included sex, age, comorbidities, and perioperative indicators. The severity of smoking was indicated by the smoking index (cigarettes/dayxyear), and respiratory diseases included chronic bronchitis, emphysema, and chronic obstructive pulmonary disease.In addition, based on previous studies, we calculated the following indicators: AFR,neutrophils-to-lymphocyte ratio(NLR), platelet-to-lymphocyte(PLR), and moncyte to lymphocyte ratio(MLR) .

\section{Statistical analysis}

Receiver operating characteristic (ROC) curve analysis using Youden's Index was established for determining the predictive value and cut-off threshold of AFR for postoperative pneumonia.Baseline data were divided into two groups with and without pneumonia, in which variables are expressed as means \pm standard deviation (SD) or numbers with percentage $(n, \%)$. Continuous variables were compared using 
Mann-Whitney U test or Student t test, whereas categorical variables using Chi-square test or Fisher exact test as appropriate,and data with statistically significant differences were included in univariate and multivariate logistic analysis.All the probabilities are double-tailed probabilities. When $P$ value is less than 0.05, there is a statistical difference.All statistical analyses were performed by SPSS version 26 (IBM, Armonk, NY, USA).

\section{Result}

\section{Baseline data of patients}

Baseline information and perioperative data of all 177 patients were listed in Table 1.The average age was 64 , with 46 females (26\%) and 131 males (74\%).According to whether the patients had postoperative pneumonia, they were divided into the pneumonia group and the non-pneumonia group, of which 32 (18\%) were in the pneumonia group. The results showed that patients with previous respiratory diseases were more likely to develop postoperative pneumonia $(P<0.05)$. Other data such as gender, age, $B M I$, comorbidities except respiratory diseases, neoadjuvant chemotherapy『length of hospital stay, ASA classification, surgical method, anesthesia intubation method, anastomotic site, intraoperative blood loss, fluid infusion, operative time, tumor location, length, and degree of differentiation were not statistically significant $(P>0.05)$.

\section{Table 1 Demographic and clinical characteristics}




\begin{tabular}{|c|c|c|c|}
\hline \multirow[t]{2}{*}{ Parameters } & \multicolumn{2}{|c|}{ Postoperative pneumonia } & \multirow[t]{2}{*}{$P$ value } \\
\hline & $Y E S \bigotimes n=32 \rrbracket$ & $\mathrm{NO} \rrbracket n=145 \rrbracket$ & \\
\hline Gender,n (\%) & & & $P=0.140$ \\
\hline Male & $27 \rrbracket 84.4 \rrbracket$ & 104囚71.7区 & - \\
\hline Female & $5 \rrbracket 15.6 \rrbracket$ & $41 \llbracket 28.3 \rrbracket$ & - \\
\hline Age(year) & $65.5 \pm 8.4$ & $63.7 \pm 8.0$ & $P=0.259$ \\
\hline $\mathrm{BMI}\left(\mathrm{kg} / \mathrm{m}^{2}\right)$ & $23.3 \pm 3.2$ & $24.0 \pm 3.0$ & $P=0.235$ \\
\hline \multicolumn{4}{|l|}{ Comorbidities, n (\%) } \\
\hline Hypertension & $14 \llbracket 43.8 \rrbracket$ & $47 \rrbracket 32.4 \rrbracket$ & $P=0.220$ \\
\hline Cerebral infarction & $4 \rrbracket 12.5 \rrbracket$ & $14 \rrbracket 14.7 \rrbracket$ & $P=0.746$ \\
\hline Coronary heart disease & $3 \rrbracket 9.4 \rrbracket$ & $19 \rrbracket 13.1 \rrbracket$ & $P=0.769$ \\
\hline Respiratory diseases & $5 \llbracket 15.6 \rrbracket$ & $4 \rrbracket 2.8 \rrbracket$ & $P=0.011$ \\
\hline Hepatitis & $2 \varangle 6.3 \rrbracket$ & $4 \rrbracket 2.8 \rrbracket$ & $P=0.297$ \\
\hline Diabetes & $4 \llbracket 12.5 \rrbracket$ & $19 \otimes 13.1 \rrbracket$ & $P=1.000$ \\
\hline The history of drinking,n (\%) & & & $P=0.637$ \\
\hline No & $23 \bigotimes 71.9 \rrbracket$ & $98 \llbracket 67.6 \rrbracket$ & - \\
\hline Yes & $9 \rrbracket 28.1 \rrbracket$ & $47 \rrbracket 32.4 \rrbracket$ & - \\
\hline Smoking index & $317 \pm 453.1$ & $250.1 \pm 429.0$ & $P=0.425$ \\
\hline History of chest surgery,n (\%) & $1 \otimes 3.1 \rrbracket$ & $5 \rrbracket 3.4 \rrbracket$ & $P=1.000$ \\
\hline The length of hospital stay(day) & $18.3 \pm 8.1$ & $17.3 \pm 5.4$ & $P=0.895$ \\
\hline Neoadjuvant chemotherapy,n (\%) & & & $P=0.071$ \\
\hline Yes & $13 \rrbracket 40.6 \rrbracket$ & $36 \rrbracket 24.8 \rrbracket$ & \\
\hline No & $19 \llbracket 59.4 \rrbracket$ & 109ه75.2》 & \\
\hline ASA Grade,n (\%) & & & $P=0.316$ \\
\hline 口 & $2 \varangle 6.2 \rrbracket$ & $2 \rrbracket 1.4 \rrbracket$ & - \\
\hline 口 & $22 \llbracket 68.8 \rrbracket$ & $125 \llbracket 86.2 \rrbracket$ & - \\
\hline 口 & $8 \otimes 25.0 \rrbracket$ & $18 \rrbracket 12.4 \rrbracket$ & - \\
\hline Surgical method,n (\%) & & & $P=0.553$ \\
\hline MIE & $27 \rrbracket 84.4 \rrbracket$ & 142ه97.9凶 & \\
\hline
\end{tabular}




\begin{tabular}{|c|c|c|c|}
\hline Open & $5 \rrbracket 15.6 \rrbracket$ & $3 \llbracket 2.1 \rrbracket$ & \\
\hline Intubation method,n (\%) & & & $P=1.000$ \\
\hline Single & 1区3.1区 & $5 \llbracket 3.4 \rrbracket$ & - \\
\hline Double & $31 \varangle 96.9 \rrbracket$ & $140 \otimes 96.6 \rrbracket$ & - \\
\hline Anastomotic site,n (\%) & & & $P=0.328$ \\
\hline Neck & $27 \rrbracket 84.4 \rrbracket$ & $132 \bowtie 91.0 \rrbracket$ & - \\
\hline Chest & $5 \rrbracket 15.6 \rrbracket$ & 13凶9.0区 & - \\
\hline Blood loss(ml) & $192.2 \pm 44.2$ & $182.3 \pm 68.7$ & $P=0.309$ \\
\hline Intraoperative fluid utilization(ml) & $2348.4 \pm 619.0$ & $2303.5 \pm 664.5$ & $P=0.715$ \\
\hline Operative time(min) & $317.4 \pm 77.6$ & $362.3 \pm 70.0$ & $P=0.546$ \\
\hline Tumor location,n (\%) & & & $P=0.447$ \\
\hline Upper & $3 \otimes 9.4 \rrbracket$ & 11区7.6区 & - \\
\hline Middle & $12 \rrbracket 37.5 \rrbracket$ & 72ه49.7凹 & - \\
\hline Lower & $17 \rrbracket 53.2 \rrbracket$ & $62 \rrbracket 42.8 \rrbracket$ & - \\
\hline Tumor length(cm) & & & $P=0.975$ \\
\hline 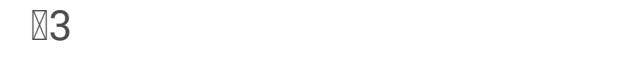 & $14 \rrbracket 43.8 \rrbracket$ & $63 \rrbracket 43.4 \rrbracket$ & - \\
\hline$\geq 3$ & $18 \rrbracket 56.3 \rrbracket$ & $82 \rrbracket 56.6 \rrbracket$ & - \\
\hline Differentiation,n (\%) & & & $P=0.159$ \\
\hline Low & $7 \rrbracket 21.9 \rrbracket$ & $32 \rrbracket 22.1 \rrbracket$ & - \\
\hline Middle & 23凶71.9凶 & $84 \rrbracket 57.9 \rrbracket$ & - \\
\hline High & $2 \otimes 6.3 \rrbracket$ & $29 \llbracket 20 \rrbracket$ & - \\
\hline
\end{tabular}

Notes:P-values were calculated by Chi-square test, Fisher exact test, Mann-Whitney U or t test Abbreviations:BMIBody mass index,Smoking index cigarettes/dayxyear,ASA American Society of Anesthesiologists

\section{Laboratory tests data}

Preoperative laboratory tests of patients with and without pneumonia were shown in Table 2. Creatinine, albumin, white blood cells, platelets, central granulophils, monocytes, fibrinogen, AFR, PLR were 
statistically different between the two groups $(P<0.05)$, and the $P$ values of fibrinogen and AFR were < 0.001 .

\section{Table 2 Laboratory tests}

\begin{tabular}{|c|c|c|c|}
\hline \multirow[t]{2}{*}{ Laboratory test } & \multicolumn{2}{|c|}{ Postoperative pneumonia } & \multirow[t]{2}{*}{$P$ value } \\
\hline & YES & NO & \\
\hline Creatinine $(\mu \mathrm{mol} / \mathrm{L})$ & $76.3 \pm 16.2$ & $71.28 \pm 16.3$ & $P=0.037$ \\
\hline $\operatorname{Albumin}(\mathrm{g} / \mathrm{L})$ & $39.1 \pm 5.1$ & $41.8 \pm 3.3$ & $P=0.005$ \\
\hline Globulin(g/L) & $26.8 \pm 4.8$ & $25.5 \pm 4.3$ & $P=0.151$ \\
\hline $\operatorname{WBC}\left(\times 10^{9} / \mathrm{L}\right)$ & $7.2 \pm 3.1$ & $5.8 \pm 1.8$ & $P=0.018$ \\
\hline Hemoglobin(g/L) & $126.1 \pm 18.7$ & $129.3 \pm 15.1$ & $P=0.3$ \\
\hline Platelet(×109) & $230.3 \pm 58.4$ & $200.4 \pm 6.6$ & $P=0.016$ \\
\hline Neutrophils $\left(\times 10^{9} / \mathrm{L}\right)$ & $4.6 \pm 2.9$ & $4.2 \pm 6.6$ & $P=0.004$ \\
\hline Lymphocyte $\left(\times 10^{9} / \mathrm{L}\right)$ & $1.8 \pm 0.7$ & $1.7 \pm 0.5$ & $P=0.690$ \\
\hline Monocytes(×109/L) & $0.6 \pm 0.2$ & $0.5 \pm 0.1$ & $P=0.015$ \\
\hline Fibrinogen(mg/dL) & $3.9 \pm 0.8$ & $3.2 \pm 0.7$ & $\mathrm{P} \otimes 0.001$ \\
\hline AFR & $10.6 \pm 3.2$ & $13.6 \pm 3.0$ & $\mathrm{P} \otimes 0.001$ \\
\hline NLR & $3.1 \pm 2.8$ & $2.7 \pm 3.7$ & $P=0.1$ \\
\hline PLR & $144.6 \pm 56.4$ & $126.8 \pm 58.8$ & $P=0.048$ \\
\hline MLR & $0.72 \pm 2.1$ & $0.3 \pm 0.2$ & $P=0.103$ \\
\hline
\end{tabular}

Notes:P-values were calculated by Mann-Whitney U or t test

Abbreviations:WBC White blood cell,AFR Albumin-to-fibrinogen ratio,NLR Neutrophils-to-Lymphocyte ratio, $P L R$ Platelet-to-Lymphocyte ratio,MLR Monocytes-Lymphocyte ratio

\section{The predictive value of AFR for postoperative pneumonia}

ROC curve analysis was used to assess the predictive value of AFR for postoperative pneumonia and the cut-off value of statistically significant laboratory test results.As shown in Figure 1, preoperative AFR level predicted postoperative pneumonia with an AUC area of 0.767 , a sensitivity of $65.6 \%$, and a specificity of 83.4\%.The cut-off values of AFR, creatinine and albumin were obtained based on the Youden's Index.Then, according to the cut-off value, all patients were divided into two groups: high level group and low level group. 


\section{Risk factors for postoperative pneumonia}

All possible risk factors $(P<0.05)$ were included in univariate and multivariate logistic regression analysis.Variables with $\mathrm{P}<0.1$ in univariate analysis were included in multivariate analysis. Multivariate logistic regression analysis showed that low creatinine, albumin and high AFR levels were independent risk factors for postoperative pneumonia in patients after radical esophagectomy.

\section{Table 3 Risk factors by univariate and multivariate logistic regression analyses}

\begin{tabular}{|c|c|c|c|c|}
\hline \multirow[t]{2}{*}{ Variables } & \multicolumn{2}{|l|}{ Univariate } & \multicolumn{2}{|l|}{ Multivariate } \\
\hline & $\mathrm{OR} \otimes 95 \% \mathrm{Cl} \bigotimes$ & $P$ value & $\mathrm{OR} \otimes 95 \% \mathrm{Cl} \mathbb{}$ & $P$ value \\
\hline Respiratory diseases (yes vs no) & $1.646-25.889$ & 0.008 & & \\
\hline Creatinine (high vs low) & $1.219-6.499$ & 0.015 & $0.081-0.712$ & 0.01 \\
\hline Albumin (high vs low) & $0.07-0.366$ & $<0.001$ & $1.268-11.656$ & 0.017 \\
\hline WBC (high vs low) & $1.678-8.190$ & 0.001 & & \\
\hline Platelet(high vs low) & $1.426-6.928$ & 0.005 & & \\
\hline Neutrophils (high vs low) & $1.906-12.633$ & 0.001 & & \\
\hline Monocytes (high vs low) & $1.738-9.392$ & 0.001 & & \\
\hline Fibrinogen (high vs low) & $2.201-11.140$ & $\llbracket 0.001$ & & \\
\hline AFR (high vs low) & $0.044-0.243$ & $\llbracket 0.001$ & $1.350-14.469$ & 0.014 \\
\hline PLR (high vs low) & $1.180-7.135$ & 0.02 & & \\
\hline
\end{tabular}

Notes:The high vs low levels were categorized using the cut-off value based on the Youden's Index

Abbreviations: WBC White blood cell, $A F R$ Albumin-to-fibrinogen ratio, $P L R$ Platelet-to-Lymphocyte ratio, $O R$ Odds ratio, $C /$ Confidence interval

\section{Discussion}

With the development of surgical techniques, postoperative complications of esophageal cancer have been significantly reduced(4). Unfortunately, surgically related complications still occur in more than half of patients with esophageal cancer (21), with pneumonia and other pulmonary complications playing an important role in the morbidity and mortality of patients undergoing esophagectomy.Postoperative pneumonia increased the postoperative mortality rate by nearly $10 \%$ and the 5 -year overall survival rate by $12 \%$ (22).Although the incidence of postoperative pneumonia can be reduced by using minimally invasive surgical techniques, persuading patients to quit smoking before surgery, exercising respiratory related muscles before surgery, and relieving postoperative wound pain (23), there is still a lack of a 
biological model that can effectively predict the incidence of postoperative pneumonia in patients.In this study, we finally found that the value of preoperative creatinine, albumin and AFR were independent influencing factors for postoperative pneumonia in patients with esophageal cancer through univariate and multivariate logistic analysis. Through ROC curve analysis, it was found that preoperative AFR value could well predict whether patients with esophageal cancer would develop pneumonia after surgery. The AUC value was 0.767 , which was the highest among the collected laboratory tests data with good sensitivity (65.6\%) and specificity (83.4\%).

In previous studies, the definition of pneumonia has been uncertain. Of the 56 studies included in a metaanalysis of postoperative complications, 18 defined pneumonia, but 16 of those definitions were different (24), so the reported incidence of pneumonia ranged from $2 \%$ to $39 \%$.In this study,we used rUPS to define postoperative pneumonia and found that 32 out of 177 patients (18\%) developed postoperative pneumonia, and patients with respiratory disease appeared to be more susceptible to postoperative pneumonia, which was consistent with the results of the two studies (25.5).To our surprise, although a growing body of evidence indicates that age is pancreatectomy, laparoscopic gastrectomy, robot assisted lung resection and other operation types of independent risk factor for postoperative complications (17), but this study (5) and another group of research did not find same age differences between two groups of patients, this could be due to different sample sizes,types of surgery and perioperative management.Since only 8 open surgeries were included, it is not reliable that there was no statistically significant difference between the two groups. In addition, a new independent factor, creatinine, was identified by univariate and multivariate logistic analysis.Creatinine as a blood indicator for evaluating renal function has been found to be an independent influence factor for postoperative acute kidney injury (AKI) in patients with esophageal cancer(26).A study included 24233 patients found high creatinine is a independent risk factor for esophageal cancer surgery related death(27),but Its association with postoperative pulmonary complications has not been studied to our knowledge. Whether preoperative serum creatinine can be used as a new marker to predict postoperative pulmonary complications and the specific mechanism in patients with esophageal cancer needs to be explored in larger sample clinical trials and basic experiments.

As mentioned above, many studies have been conducted on albumin and AFR as independent influencing factors to predict postoperative complications and prognosis in tumor patients.In this study, albumin levels were found to be statistically different between the two groups, consistent with previous findings (12) and associated with postoperative complications in a study on the ratio of C-reactive protein to albumin (15).From the results, increasing albumin levels to a certain extent can prevent postoperative complications, which is correlated with previous reports that low serum albumin may lead to a higher risk of worsening disease and poor prognosis in cancer patients. This might be due to malnutrition can weaken the immune system, increase the chance of infection, and albumin may help stabilize the DNA replication and cell growth, regulate the body's reaction, enhance immunity, prevention of malignant disease (18). 
AFR, as a new indicator, represents the combined effect of two blood factors, enhancing sensitivity to assess inflammation and nutritional status. In various models of acute ST-segment elevation myocardial infarction (STEMI) (28),non small cell lung cancer(NSCLC)(29), soft tissue sarcoma (30), and esophageal cancer (18), the combination of albumin and fibrinogen has been reported to be superior to albumin and fibrinogen alone, and has been widely recommended as a prognostic factor.lt should be noted that in a report on the prediction of prognosis of esophageal cancer by AFR (18), postoperative complications of patients were not included in the study, so it was not possible to assess whether the prognosis of patients after esophageal cancer was caused by the occurrence of postoperative complications. However, it remains to be further explored whether AFR or postoperative pneumonia has a stronger correlation with prognosis. To date, this study is the first retrospective study of the relationship between AFR and postoperative pneumonia in patients with esophageal cancer.

However, there are still several deficiencies in our study.First of all, this study is a retrospective, singlecenter study, which is likely to cause selective bias, and it needs to be verified by prospective, multicenters,large-sample trials in the future.In addition, it is not clear whether preoperative intervention to change AFR level can reduce the incidence of postoperative pneumonia in patients with esophageal cancer.Finally, the mechanism that how AFR can predict postoperative pneumonia in patients with esophageal cancer is still unclear, and the molecular mechanism may be found through relevant basic experiments.

\section{Conclusion}

First of all, this study found that preoperative AFR can effectively predict the occurrence of postoperative pneumonia in patients with esophageal cancer as an independent influence factor. Secondly, creatinine, as another independent influence factor, may provide a new direction for research in this field in the future.

\section{Abbreviations}

MIE:minimally invasive esophagectomy;RCT:randomized controlled trial;CT:computed tomography;AFR: Albumin-to-fibrinogen ratio;rUPS:the revised Uniform Pneumonia Score;ASA: American Society of Anesthesiologists;NLR:neutrophils-to-lymphocyte ratio;PLR:platelet-to-lymphocyte;MLR:moncyte-tolymphocyte ratio;BMl:Body mass index; Cl: Confidence interval;OR: Odds ratio;WBC: White blood cell;AKI:acute kidney injury;STEMI: ST-segment elevation myocardial infarction;NSCLC:non small cell lung cancer.

\section{Declarations}

\section{Ethics approval and consent to participate}

This retrospective study was approved by the The Ethics Committee of Scientific Research and Clinical Trial of The First Affiliated Hospital of Zhengzhou University, and the requirement for informed patient 
consent was waived due to the retrospective nature of this study.

\section{Consent for publication}

Not applicable

\section{Availability of data and materials}

The datasets used and analyzed during the current study are available from the corresponding author on reasonable request.

\section{Competing interests}

The authors declare that they have no competing interests

\section{Funding}

This study received no external funding

\section{Authors' contributions}

Writing, review, Zhang Peng; editing, Zhao Song

\section{Acknowledgements}

Not applicable

\section{References}

1. Wong MCS. Hamilton W, Whiteman DC, et al Global Incidence and mortality of oesophageal cancer and their correlation with socioeconomic indicators temporal patterns and trends in 41 countries. Sci Rep. 2018;8(1):4522.

2. Bray F. Ferlay J, Soerjomataram I. Siegel RL, Torre LA. Jemal A. Global cancer statistics 2018 : GLOBOCAN estimates of incidence and mortality worldwide for 36 cancers in 185 countries [published correction appears in CA Cancer J Clin. 2020 Jul;70(4):313]. CA Cancer J Clin. 2018;68(6):394-424..

3. Yang H. Liu H, Chen Y, et al Neoadjuvant Chemoradiotherapy Followed by Surgery Versus Surgery Alone for Locally Advanced Squamous Cell Carcinoma of the Esophagus (NEOCRTEC5010): A Phase III Multicenter, Randomized, Open-Label Clinical Trial. J Clin Oncol. 2018;36(27):2796-803.

4. Khan O. Nizar S, Vasilikostas G. Wan A. Minimally invasive versus open oesophagectomy for patients with oesophageal cancer: a multicentre, open-label, randomised controlled trial. J Thorac Dis. 2012;4(5):465-6.

5. Kanda M. Koike M, Tanaka C, et al Risk Prediction of Postoperative Pneumonia After Subtotal Esophagectomy Based on Preoperative Serum Cholinesterase Concentrations. Ann Surg Oncol. 
2019;26(11):3718-26.

6. Kataoka K. Takeuchi H, Mizusawa J, et al Prognostic Impact of Postoperative Morbidity After Esophagectomy for Esophageal Cancer: Exploratory Analysis of JCOG9907. Ann Surg. 2017;265(6):1152-7.

7. Nozoe T. Kimura Y, Ishida M. Saeki H, Korenaga D. Sugimachi K. Correlation of pre-operative nutritional condition with post-operative complications in surgical treatment for oesophageal carcinoma. Eur J Surg Oncol. 2002;28(4):396-400.

8. Hashimoto S. Tominaga T, Nonaka T, et al The C-reactive protein to albumin ratio predicts postoperative complications in oldest-old patients with colorectal cancer. Int $\mathrm{J}$ Colorectal Dis. 2020;35(3):423-31.

9. Kijima T. Arigami T, Uchikado Y, et al Combined fibrinogen and neutrophil-lymphocyte ratio as a prognostic marker of advanced esophageal squamous cell carcinoma. Cancer Sci. 2017;108(2):193-9.

10. Kurita D. Oguma J, Ishiyama K. Hirano Y, Kanamori J. Daiko H. Handgrip Strength Predicts Postoperative Pneumonia After Thoracoscopic-Laparoscopic Esophagectomy for Patients with Esophageal Cancer. Ann Surg Oncol. 2020;27(9):3173-81.

11. Goh SL. De Silva RP, Dhital K, Gett RM. Is low serum albumin associated with postoperative complications in patients undergoing oesophagectomy for oesophageal malignancies? Interact Cardiovasc Thorac Surg. 2015;20(1):107-13.

12. Lin MY. Liu WY, Tolan AM. Aboulian A, Petrie BA. Stabile BE. Preoperative serum albumin but not prealbumin is an excellent predictor of postoperative complications and mortality in patients with gastrointestinal cancer. Am Surg. 2011;77(10):1286-9.

13. Quhal F. Pradere B, Sari Motlagh R, et al. Prognostic value of preoperative albumin to globulin ratio in patients treated with salvage radical prostatectomy for radiation recurrent prostate cancer. Minerva Urol Nefrol.2020;10.23736/S0393-2249.20.03938-7..

14. Baik H. Bae KB. Low albumin level and longer interval to closure increase the early complications after ileostomy closure. Asian J Surg. 2020;S1015-9584(20)30282-7.

15. Yasukawa K. Shimizu A, Motoyama H, et al. Preoperative C-reactive protein-to-albumin ratio predicts long-term outcomes in extrahepatic cholangiocarcinoma patients. J Surg Oncol. 2020;10.1002/jso.26109.

16. Guan X. Gong M, Wang X, et al Low preoperative fibrinogen level is risk factor for neurological complications in acute aortic dissection. Med (Baltim). 2018;97(21):e10830.

17. You X. Zhou Q, Song J. Gan L, Chen J. Shen H. Preoperative albumin-to-fibrinogen ratio predicts severe postoperative complications in elderly gastric cancer subjects after radical laparoscopic gastrectomy. BMC Cancer. 2019;19(1):931.

18. Tan Z. Zhang M, Han Q, et al A novel blood tool of cancer prognosis in esophageal squamous cell carcinoma: the Fibrinogen/Albumin Ratio. J Cancer. 2017;8(6):1025-9. 
19. Song YN. Qi Y, Zhang CY, et al A new technology for reducing anastomotic fistula in the neck after esophageal cancer surgery. J Thorac Dis. 2019;11(7):3084-92.

20. Weijs TJ. Seesing MF, van Rossum PS, et al. Internal and External Validation of a multivariable Model to Define Hospital-Acquired Pneumonia After Esophagectomy. J Gastrointest Surg. 2016;20(4):6807.

21. Low DE. Kuppusamy MK, Alderson D, et al Benchmarking Complications Associated with Esophagectomy. Ann Surg. 2019;269(2):291-8.

22. Booka E. Takeuchi H, Nishi T, et al. The Impact of Postoperative Complications on Survivals After Esophagectomy for Esophageal Cancer [published correction appears in Medicine (Baltimore). 2015 Sep;94(39):1]. Medicine (Baltimore). 2015;94(33):e1369..

23. Chevallay M. Jung M, Chon SH. Takeda FR, Akiyama J. Mönig S. Esophageal cancer surgery: review of complications and their management [published online ahead of print, 2020 Sep 15]. Ann NY Acad Sci. 2020;10.1111/nyas. 14492..

24. Blencowe NS. Strong S, McNair AG, et al Reporting of short-term clinical outcomes after esophagectomy: a systematic review. Ann Surg. 2012;255(4):658-66.

25. Wei R. Dong W, Shen $\mathrm{H}$, et al Predictive Effects of Lung function test on Postoperative Pneumonia in Squamous Esophageal Cancer. Sci Rep. 2016;6:23636.

26. Wang W. Wang T, Feng X. Sun L. Incidence and risk factors of acute kidney injury after esophageal cancer surgery: A nested case-control study. Int J Surg. 2017;39:11-5.

27. Yoshida N. Yamamoto H, Baba H, et al Can Minimally Invasive Esophagectomy Replace Open Esophagectomy for Esophageal Cancer? Latest Analysis of 24,233 Esophagectomies From the Japanese National Clinical Database. Ann Surg. 2020;272(1):118-24.

28. Zhao Y. Yang J, Ji Y, et al Usefulness of fibrinogen-to-albumin ratio to predict no-reflow and shortterm prognosis in patients with ST-segment elevation myocardial infarction undergoing primary percutaneous coronary intervention. Heart Vessels. 2019;34(10):1600-7.

29. Ying J. Zhou D, Gu T. Huang J, Liu H. Pretreatment albumin/fibrinogen ratio as a promising predictor for the survival of advanced non small-cell lung cancer patients undergoing first-line platinum-based chemotherapy. BMC Cancer. 2019;19(1):288.

30. Liang Y. Wang W, Que Y, et al Prognostic value of the fibrinogen/albumin ratio (FAR) in patients with operable soft tissue sarcoma. BMC Cancer. 2018;18(1):942.

\section{Figures}




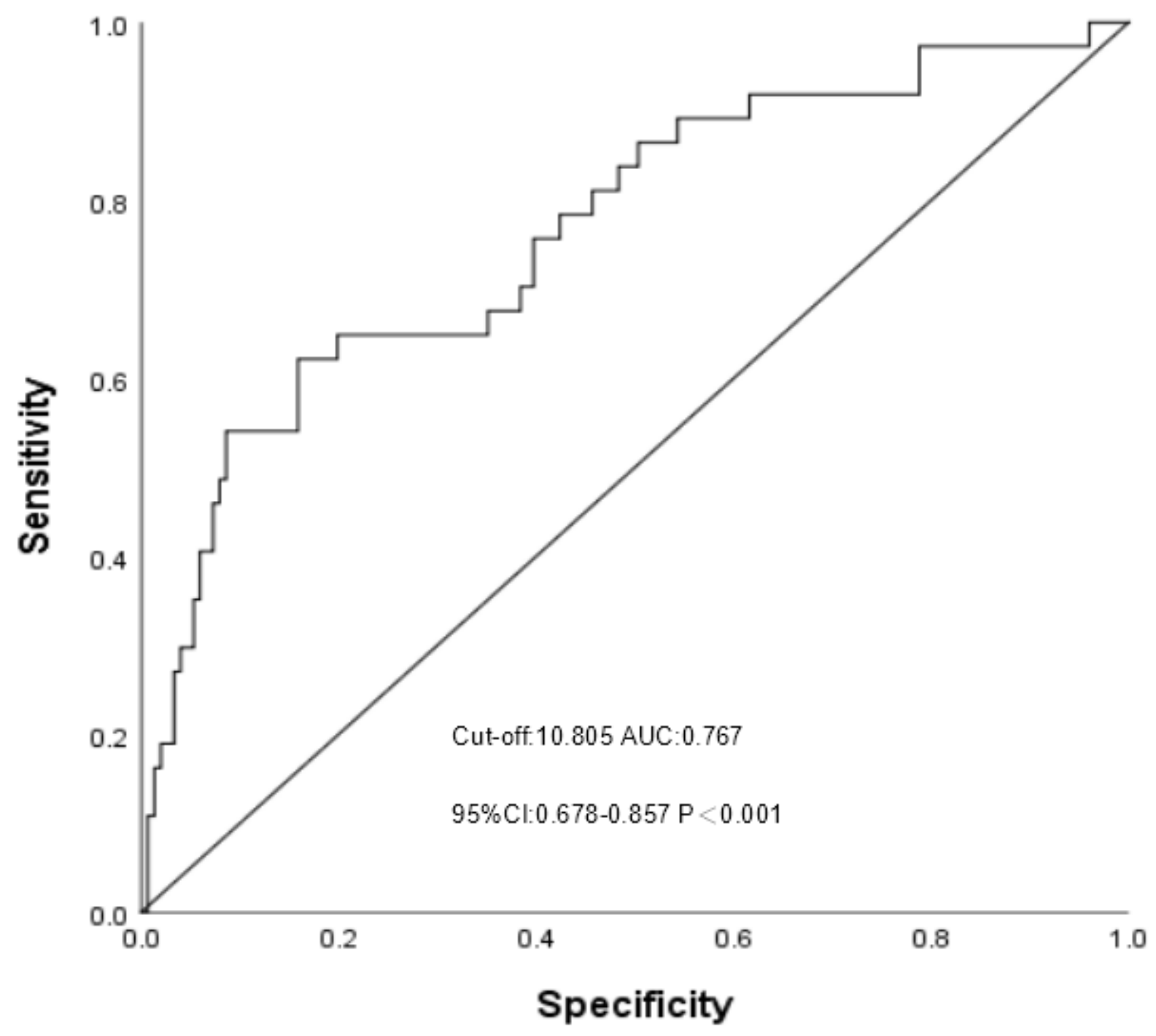

Figure 1

ROC curve analysis of AFR for postoperative pneumonia Notes:Predictive value of AFR for postoperative pneumonia by ROC curve analysis. The results indicated preoperative AFR as a potential predictive factor with an AUC of $0.767,95 \% \mathrm{Cl}$ of $0.678-0.857$, a cut-off value of 10.805 , a sensitivity of $65.6 \%$ and a specificity of $83.4 \%$, respectively $(P<0.001)$. Abbreviations:AFR, albumin-to-fibrinogen ratio; ROC, receiver operating characteristic; $\mathrm{AUC}$, the area under the curve; $\mathrm{Cl}$, confidence interval 